\title{
Tackling disparities in achievement of optimal functioning: delivering health services to meet the needs of ethnic minorities
}

\author{
Sarah Carne \\ Accident Compensation Corporation, New Zealand. sarah.carne@acc.co.nz
}

Background: Despite the utilisation of best practice when delivering services, disparities in outcomes exist across ethnic groups. Access to and uptake of services is considered to contribute to these disparities which inhibits the ability of people with disabilities to participate in life to fullest extent. Objective: To investigate barriers experienced by minority cultures when accessing health services and ways in which these barriers can be overcome. Methods: Interviews with clients with spinal cord impairment who belong to minority ethnic groups, service providers and sector experts.

Findings: Multiple barriers exist when accessing services; they include language, cultural characteristics and views of disability and appropriateness of services. Culture can have an effect on the whole continuum of care from how the disability is interpreted by the individual and/or family, how they respond to care, their understanding, communication of needs, engagement with the process, and the opportunities to re-connect and participate within their communities. Community providers are aware of the needs of their clients and have strategies to overcome these barriers, however they work in isolation from funding bodies and other organisations and there is a lack of a framework or model that can guide and inform organisations and providers.

Discussion: While the research identified a multitude of barriers that can impede access to and uptake of services to people belonging to minority ethnicities, community knowledge can be utilised to overcome a significant number of barriers.

Conclusion: A number of recommendations are made to improve access to and uptake of health services including a strategic approach, interagency collaboration, cultural competency training, cultural brokering and disability awareness within the community. 